\title{
Melittin inhibits osteoclast formation through the downregulation of the RANKL-RANK signaling pathway and the inhibition of interleukin-1 $\beta$ in murine macrophages
}

\author{
JUNG-YOON CHOE and SEONG-KYU KIM
}

\author{
Division of Rheumatology, Department of Internal Medicine, Arthritis and Autoimmunity Research Center, \\ Catholic University of Daegu School of Medicine, Daegu, Republic of Korea
}

Received November 25, 2015; Accepted December 23, 2016

DOI: $10.3892 /$ ijmm.2017.2876

\begin{abstract}
Melittin is a major toxic component of bee venom (Apis mellifera). It is not known whether melittin is involved in bone metabolism and osteoclastogenesis. The aim of this study was to determine the role of melittin in the regulation of osteoclastogenesis. In vitro osteoclastogenesis assays were performed using mouse RAW 264.7 cells and bone marrowderived macrophages (BMMs) treated with receptor activator of nuclear factor- $\kappa \mathrm{B}$ ligand (RANKL) and macrophage colonystimulating factor (M-CSF). Morphologic and functional analyses for osteoclast-like multinucleated cells (MNCs) were performed by tartrate-resistant acid phosphatase (TRAP) staining, F-actin staining and pit formation methods. The gene expression of TRAP, cathepsin K, matrix metalloproteinase-9 (MMP-9) and carbonic anhydrase II was measured by reverse transcription-quantitative PCR. The protein expression levels of mitogen-activated protein kinases (MAPKs), the p65 subunit of nuclear factor- $\kappa \mathrm{B}(\mathrm{NF}-\kappa \mathrm{B}), \mathrm{c}-\mathrm{Fos}, \mathrm{c}-\mathrm{Jun}$, nuclear factor of activated T cells, cytoplasmic 1 (NFATc1), TNF receptorassociated factor-6 (TRAF6), and interleukin-1 $\beta$ (IL-1 $\beta$ ) were assessed by western blot analysis. Melittin inhibited the mRNA expression of TRAP, cathepsin K, MMP-9 and carbonic anhydrase II in RANKL-stimulated RAW 264.7 cells. The increased protein expression of TRAF6, p-extracellular signal-regulated kinase (ERK), p-JNK, p-p65, p-c-Fos and NFATc1 induced by RANKL was significantly suppressed in the RAW 264.7 cells treated with melittin. A synergistic effect of IL-1 $\beta$ on the formation of RANKL-induced osteoclast-like MNCs was found in two experimental cells. The increased expression
\end{abstract}

Correspondence to: Dr Seong-Kyu Kim, Division of Rheumatology, Department of Internal Medicine, Arthritis and Autoimmunity Research Center, Catholic University of Daegu School of Medicine, 33 Duryugongwon-ro 17-gil, Nam-gu, Daegu 705-718, Republic of Korea

E-mail: kimsk714@cu.ac.kr

Key words: melittin, osteoclast, interleukin-1 $\beta$, receptor activator of nuclear factor- $\kappa \mathrm{B}$ ligand, mitogen-activated protein kinase, nuclear factor of activated $\mathrm{T}$ cells, cytoplasmic 1 of IL-1 $\beta$ following the stimulation of RAW 264.7 cells with RANKL activated TRAF6, p-ERK, p-JNK, p-p65, p-c-Fos and NFATc1. These effects were attenuated by the downregulation of IL-1 $\beta$ using siRNA against IL-1 $\beta$, and also by treatment with melittin. On the whole, the findings of this study demonstrate that melittin inhibits the formation of osteoclast-like MNCs by interfering with the RANKL-RANK signaling pathway.

\section{Introduction}

Osteoclasts, mainly responsible for bone resorption, are multinucleated giant cells formed by the fusion of precursor cells of myeloid-originated hematopoietic monocyte/macrophage lineage (1-3). The receptor activator of nuclear factor- $\kappa \mathrm{B}(\mathrm{NF}-\kappa \mathrm{B})$ ligand (RANKL)-RANK system is a key regulator of osteoclastogenesis under physiological conditions. However, several pro-inflammatory cytokines, including tumor necrosis factor- $\alpha$ (TNF- $\alpha$ ) and interleukin (IL)-1, in combination with the RANK-RANKL complex, play a crucial role in enhancing osteoclast differentiation and activation in chronic inflammatory conditions, such as rheumatoid arthritis (RA), psoriatic arthritis (PsA), and chronic gouty arthritis (4-7). In in vitro studies, IL-1 has been found to be a pluripotent cytokine that exhibits bone resorption activity through interaction with the RANKL-RANK system or binding with the IL-1 receptor (IL-1R) (8-10). Under the aegis of the p38 mitogen-activated protein kinase (MAPK), IL-1 mediates TNF- $\alpha$-induced RANKL expression by stromal cells and promotes the differentiation of osteoclast precursor cells (10). This suggests that IL-1 is a crucial cytokine for osteoclast differentiation and activation, and can thus be considered a potent therapeutic target in the treatment of bone disorders (11).

Bee venom (Apis mellifera) has been shown to have antiinflammatory and anti-nociceptive properties in human (12) and animal models $(13,14)$. Melittin is a major component of bee venom (15) and is commonly studied for its therapeutic potential in chronic inflammatory joint diseases $(14,16)$. These investigations have demonstrated a distinct anti-arthritic effect through the inactivation of the $\mathrm{NF}-\kappa \mathrm{B}$ signaling pathway and the induction of the apoptosis of RA synoviocytes $(14,16)$. With regard to the effect of melittin on bone metabolism, Tashiian et al reported that melittin was a potent stimulator of 
prostaglandin synthesis in bone and bone resorption, which was inhibited by indomethacin (17). By contrast, the water-soluble fraction of bee venom has been shown to significantly inhibit pathological bone changes in a rat model of arthritis (13).

To the best of our knowledge, there are limited studies available on the effects of melittin on osteoclast formation or bone resorption $(13,17)$. Thus, in the present study, we investigated whether melittin influences RANKL-induced osteoclast formation in murine RAW 264.7 cells and bone marrow-derived macrophages (BMMs). In addition, we examined the effects of melittin on IL-1 $\beta$-mediated osteoclast formation in vitro.

\section{Materials and methods}

Cell culture and osteoclast differentiation. BMMs were isolated from 6-week-old male C57BL/6 mice (Samtako Inc., Osan, Korea) as previously described (18), following the approval of the Institutional Review Board Animal Trial Board Committee of Daegu Catholic University Medical Center, Daegu, Korea. BMMs were cultured in $\alpha$-MEM containing $10 \%$ fetal bovine serum (FBS) and $10 \mathrm{ng} / \mathrm{ml}$ macrophage colony-stimulating factor (M-CSF) for $24 \mathrm{~h}$. Non-adherent cells were removed the following day and cultured in the presence of $30 \mathrm{ng} / \mathrm{ml} \mathrm{M-CSF}$ for 3 days. The attached cells were then used to generate osteoclasts from BMMs. The cells $\left(5 \times 10^{4}\right.$ cells/well) were cultured with various treatment combinations of M-CSF (30 ng/ml), RANKL (50 ng/ml) and IL-1 $\beta$ (100 ng/ml) in 24-well tissue culture plates.

The RAW 264.7 cells (Korean Cell Line Bank, Seoul, Korea) were cultured in Dulbecco's modified Eagle's medium (DMEM) supplemented with $10 \%$ FBS and $1 \%$ antibiotics. The cells were grown at $37^{\circ} \mathrm{C}, 5 \% \mathrm{CO}_{2}$ in fully humidified air. For the establishment of osteoclast differentiation, the RAW 264.7 cells were incubated at $8 \times 10^{4}$ cells/well in 6-well plates for 7 days with DMEM containing RANKL (50 ng/ml). The medium was refreshed every 2 days. Cell viability was assessed by 3-(4,5-dimethylthiazol-2-yl)-2,5-diphenyltetra zolium bromide (MTT) assay (Sigma, St. Louis, MO, USA). The RAW 264.7 cells and BMMs treated with melittin up to a concentration of $1 \mu \mathrm{g} / \mathrm{ml}$ exhibited $>80 \%$ viability after $24 \mathrm{~h}$ (Fig. 1). However, the viability of the RAW 264.7 cells and BMMs treated with melittin at $5 \mu \mathrm{g} / \mathrm{ml}$ was significantly decreased. Based on these results, the non-cytotoxic concentration of melittin $(1 \mu \mathrm{g} / \mathrm{ml})$ was used in our experiments.

Reagents and antibodies. Recombinant mouse RANKL, M-CSF and IL-1 $\beta$ were purchased from PeproTech (Rocky Hill, NJ, USA). Melittin was purchased from Sigma. Components of cell culture media were obtained as follows: DMEM (GeneDEPOT, Barker, TX, USA), $\alpha$-MEM (Gibco-BRL, Grand Island, NY, USA) and $10 \%$ FBS (HyClone, Logan, UT, USA). The antibodies used were as follows: phosphorylated (p-)c-Jun (sc-16312), nuclear factor of activated T cells, cytoplasmic 1 (NFATc1; sc-7294), TNF receptor-associated factor-6 (TRAF6; sc-7221), p-extracellular signal-regulated kinase (ERK; sc-7383), ERK (sc-94), $\beta$-actin (sc-47778) and goat anti-rabbit IgG-HRP (all from Santa Cruz Biotechnology, Inc., Santa Cruz, CA, USA), osteoprotegerin (OPG; ab73400-100), IL-1 $\beta$ (ab9722), p-p38 (ab47363) and mouse IgG-HRP (all from Abcam, Cambridge, MA, USA), RANK (AM56) and RANKL (AM57) (from Calbiochem,
San Diego, CA, USA), p-p65 subunit of NF-кB (\#3033), p-JNK (\#9252), p-c-Fos (\#5348), p38 (\#9212) and JNK (\#9252) (all from Cell Signaling Technology, Beverly, MA, USA).

Tartrate-resistant acid phosphatase (TRAP) staining. The RAW 264.7 cells were plated in a 6-well culture dish and stimulated with RANKL (50 ng/ml). After 7 days of culture, the cells were stained for TRAP using a leukocyte acid phosphatase kit (Takara Bio, Inc., Shiga, Japan) according to the manufacturer's instructions. After 5 days of culture, the BMMs were also stained for TRAP. The cultured cells were fixed in a fixation solution for $5 \mathrm{~min}$ at room temperature and washed with distilled water. TRAP-positive MNCs with at least 3 or more nuclei were defined as osteoclasts. Three random selected areas at each well were used to count the number of osteoclastlike MNCs. This procedure was repeated 3 times. The number of osteoclast-like MNCs was expressed as an average.

Pit formation assay and F-actin ring staining. The RAW 264.7 cells were plated in a 24-well culture dish and stimulated with RANKL (50 ng/ml) for 10 days. To examine bone resorption, we used a pit formation assay plate kit (Corning Inc., Corning, NY, USA). After 10 days of osteoclast culture, the conditioned medium was removed from each well and the cells were treated with $5 \%$ sodium hypochlorite for $5 \mathrm{~min}$. After washing the plate with water and allowing it to dry, regions of each well were photographed using a microscope. For F-actin ring staining, osteoclasts differentiated from RAW 264.7 cells and BMMs were cultured for 7 days. Staining was carried out using Phalloidin CruzFluor ${ }^{\mathrm{TM}} 488$ Conjugate (Santa Cruz Biotechnology, Inc.). The cells were washed with phosphatebuffered saline (PBS), fixed in 3.5\% neutral buffered formalin for $10 \mathrm{~min}$ at room temperature, and permeabilized for $10 \mathrm{~min}$ with $0.1 \%$ Triton $\mathrm{X}-100$ in PBS. The cells were blocked with $1 \%$ BSA for $1 \mathrm{~h}$ at room temperature and incubated with Phalloidin CruzFluor ${ }^{\mathrm{TM}} 488$ Conjugate (Santa Cruz, Heidelberg, Germany) (1:5,000 dilution) for $15 \mathrm{~min}$. The cells were then washed 3 times with PBS and photographed using a fluorescence microscope (Olympus, Tokyo, Japan).

Reverse transcription-quantitative polymerase chain reaction $(R T-q P C R)$ for analysis of gene expression. To evaluate the mRNA levels of TRAP, cathepsin K, matrix metalloproteinase-9 (MMP-9) and carbonic anhydrase II, the RAW 264.7 cells were stimulated with RANKL, treated with melittin and cultured for the indicated periods of time $(0,3$, 5, 7, 9 and 11 days). Total RNA was extracted using TRIzol reagent (Gibco-BRL). cDNA synthesis was performed using the GeNet Bio kit (GeNet Bio, Cheonan, Korea). Quantitative PCR amplification was performed using a RT-PCR machine (BioRad, Hercules, CA, USA) and SYBR-Green Supermix (Toyobo, Tokyo, Japan). We used the following PCR protocol: $95^{\circ} \mathrm{C}$ for $3 \mathrm{~min} ; 40$ cycles $\left(15 \mathrm{sec}, 95^{\circ} \mathrm{C} / 1 \mathrm{~min}, 58-62^{\circ} \mathrm{C}\right)$; and $72^{\circ} \mathrm{C} / 45 \mathrm{sec}$; and $60^{\circ} \mathrm{C}$ to $95^{\circ} \mathrm{C}$ per cycle for melting curve analysis. The oligonucleotide primers used in this analysis are listed in Table I.

Western blot analysis. The RAW 264.7 cells were pre-treated with melittin $(1 \mu \mathrm{g} / \mathrm{ml})$ for $2 \mathrm{~h}$ and stimulated with RANKL for the indicated periods of time. The cells were harvested after washing with PBS buffer. Total protein was extracted 

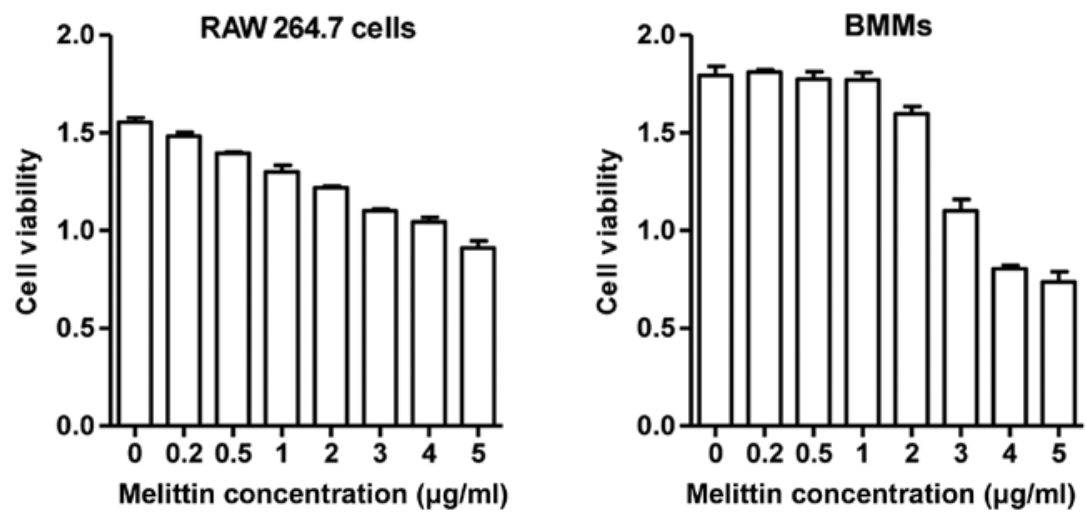

Figure 1. Cell viability assay in experimental cells including RAW 264.7 cells and bone marrow-derived macrophages (BMMs) incubated with melittin.

in an IPH buffer [1 M Tris ( $\mathrm{pH} 8.0), 5 \mathrm{M}$ sodium chloride, $10 \%$ Nonidet P-40 and protease inhibitor cocktail (Roche, Indianapolis, IN, USA)]. The protein concentration was determined using the Bio-Rad protein assay kit (Bio-Rad, Hercules, CA, USA). Cell lysates of equal protein concentrations were prepared in Laemmli protein sample (LDS) buffer (Bio-Rad). Cell lysates were separated by $10 \%$ sodium dodecyl sulfate-polyacrylamide gel electrophoresis (SDS-PAGE) gel electrophoresis and transferred onto nitrocellulose membranes (Bio-Rad). The membranes were then rinsed and blocked with 5\% non-fat dry milk in TBS $/ 0.1 \%$ Tween-20 for $1 \mathrm{~h}$, incubated with primary antibodies overnight at $4^{\circ} \mathrm{C}$, and then with a horseradish peroxidase-conjugated secondary antibody for $1 \mathrm{~h}$. Immunoreactive proteins were detected using the ELC western blot detection system kit (Amersham, Braunschweig, Germany).

Transfection with IL-1 $\beta$ siRNA. For the determination of IL-1 $\beta$-mediated osteoclast differentiation, the RAW 264.7 cells were seeded at $2 \times 10^{5}$ cells/well in 6-well plates in DMEM. The cells were transfected with mouse IL-1 $\beta$ siRNA (Invitrogen, Carlsbad, CA, USA) to a final concentration of $50 \mathrm{nM}$ using Lipofectamine RNAiMax (Invitrogen). Negative control siRNA used was non-targeting negative control siRNA (Med GC; Invitrogen). Negative control siRNA was also used to refer to 'mock-transfected cells' in the experiments. After $72 \mathrm{~h}$, the medium was replaced with differentiation medium and the cells were treated.

Statistical analysis. Statistical analysis for significant differences was performed using a non-parametric Wilcoxon signed rank sum test between two groups. Statistical significance was determined at p-values <0.05. All statistical analyses were carried out using IBM SPSS Statistics 19.0 (IBM Corp., Armonk, NY, USA).

\section{Results}

Melittin inhibits the formation of osteoclast-like MNCs. The gene expression of TRAP, cathepsin K, MMP-9 and carbonic anhydrase II gradually increased in the RAW 264.7 cells during stimulation with RANKL (50 ng/ml) (Fig. 2A). Maximal expression was observed on day 7 for TRAP and cathepsin $\mathrm{K}$ and on day 9 for MMP-9 and carbonic anhydrase II. The expression of these genes decreased thereafter. The increased
Table I. Specific primer sequences used in quantitative RT-PCR.

\begin{tabular}{ll}
\hline Gene & \multicolumn{1}{c}{ Sequence $\left(5^{\prime} \rightarrow 3^{\prime}\right)$} \\
\hline TRAP & F: AAG GCG AGA GAT TCT TTC CCT G \\
& R: ACT GGG GAC AAT TCA CTA GAG C \\
Cathepsin K & F: CAG CAG AAC GGA GGC ATT GA \\
& R: CCT TTG CCG TGG CGT TAT AC \\
MMP-9 & F: GCC CTG GAA CTC ACA CGA CA \\
& R: TTG GAA ACT CAC ACG CCA GAA G \\
Carbonic & F: CAT TAC TGT CAG CAG CGA GCA \\
anhydrase II & R: GAC GCC AGT TGT CCA CCA TC \\
IL-1 $\beta$ & F: GCC TCG TGC TGT CGG ACC \\
GAPDH & R: TGT CGT TGC TTG GTT CTC CTT G \\
& F: AAG GCT GTG GGC AAG GTC ATC \\
& R: CAG GCG GCT CAG ATC C
\end{tabular}

TRAP, tartrate-resistant acid phosphatase; MMP-9, matrix metalloproteinase-9; IL-1 $\beta$, interleukin-1 $\beta$; GAPDH, glyceraldehyde 3-phosphate dehydrogenase.

expression of these osteoclastogenic genes in the RAW 264.7 cells cultured with RANKL for 7 days was significantly inhibited by pre-treatment with melittin for 3 and 5 days (Fig. 2B). In addition, melittin (ranging from 0.2 to $1.0 \mu \mathrm{g} / \mathrm{ml}$ ) dosedependently suppressed TRAP, cathepsin K, MMP-9 and carbonic anhydrase II expression in RAW 264.7 cells treated with RANKL (50 ng/ml) (Fig. 2C).

TRAP staining and F-actin ring staining demonstrated that, for the RAW 264.7 cells, incubation with RANKL $(50 \mathrm{ng} / \mathrm{ml})$ for 7 days markedly induced the formation of TRAP $(+)$ and F-actin(+) MNCs. This was significantly attenuated by pretreatment with melittin $(1 \mu \mathrm{g} / \mathrm{ml})$ for 3 and 5 days (Fig. 2D). The pit formation assay for bone resorption revealed that stimulating the RAW 264.7 cells with RANKL for 10 days promoted the generation of numerous and variable-sized pits. The addition of melittin for 3 and 5 days prominently reduced the number and size of the pits (Fig. 2D).

Treatment of the BMMs with M-CSF (30 ng/ml) alone to failed to induce the formation of osteoclast-like cells (Fig. 2E). However, TRAP staining assay illustrated that the BMMs 

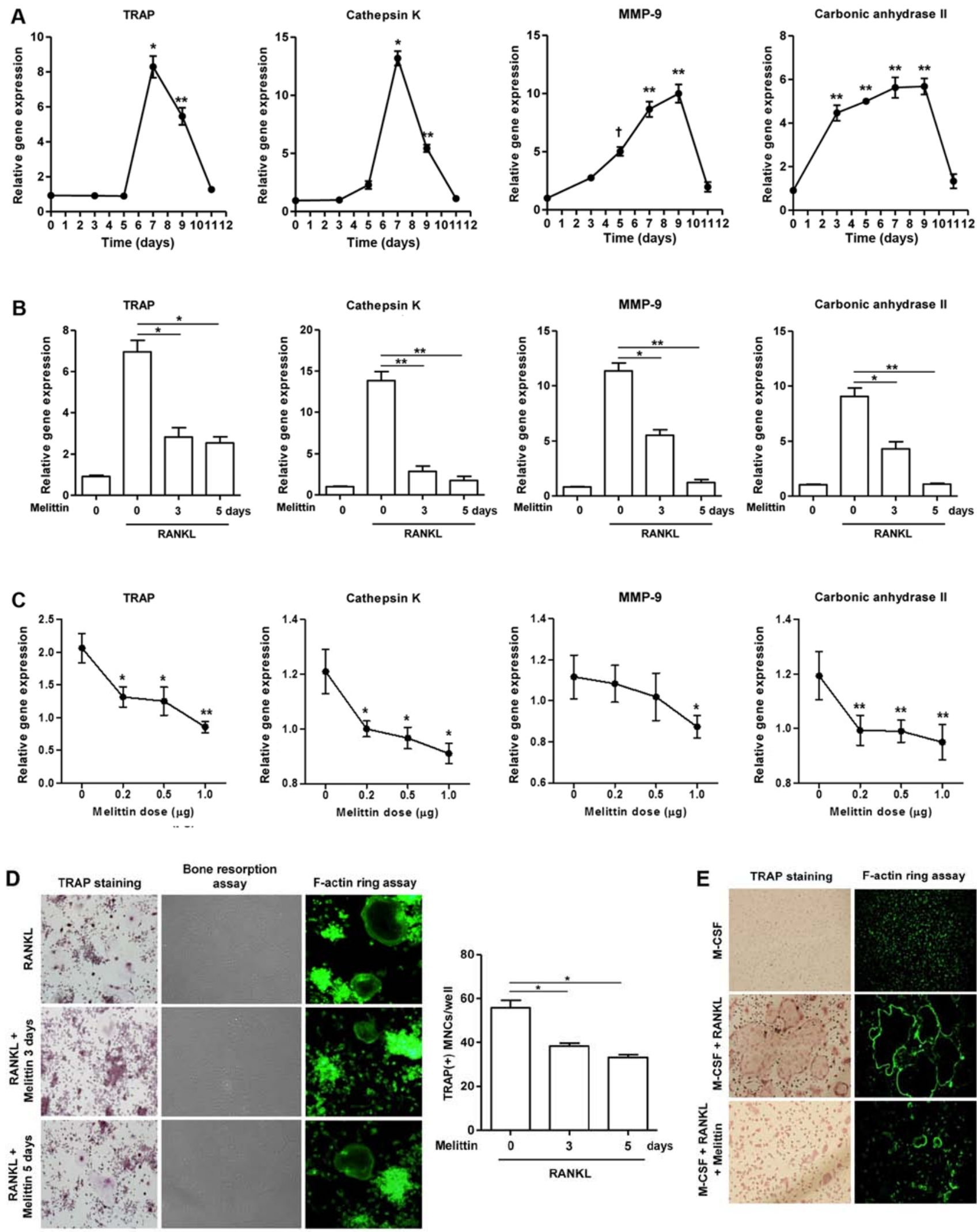

Figure 2. Melittin influencea osteoclast formation in time- and dose-dependent manner. (A) Osteoclastogenic genes, such as TRAP, cathepsin K, matrix metalloproteinase-9 (MMP-9) and carbonic anhydrase II, in the course of osteoclastogenesis in RAW 264.7 cells incubated with receptor activator of nuclear factor- $\mathrm{kB}$ ligand (RANKL) $(50 \mathrm{ng} / \mathrm{ml})$ for $0,3,5,7,9$ and 11 days were measured $\left({ }^{*} \mathrm{p}<0.001,{ }^{* *} \mathrm{p}<0.01\right.$ and ${ }^{\dagger} \mathrm{p}<0.05$ compared to non-treated cells). (B) Melittin $(1 \mu \mathrm{g} / \mathrm{ml})$ was added to the RAW 264.7 cells on days 3 and 5 following stimulation with RANKL. Inhibitory effect of melittin $(1 \mu \mathrm{g} / \mathrm{ml})$ treatment for 0,3 and 5 days on osteoclastogenic genes was assessed ("p $<0.01$ and ${ }^{* *} \mathrm{p}<0.001$ compared to RANKL-stimulated cells without melittin). (C) RAW 264.7 cells treated with RANKL ( $50 \mathrm{ng} / \mathrm{ml})$ were cultured at different dosages of melittin $(0.2$ to $1.0 \mu \mathrm{g} / \mathrm{ml})\left({ }^{*} \mathrm{p}<0.05\right.$ and ${ }^{* *} \mathrm{p}<0.01$ compared to non-treated cells). (D) Melittin was added to the RAW 264.7 cells on days 3 and/or 5 following stimulation with RANKL. The in vitro morphological and functional analyses for osteoclastogenesis were performed using TRAP staining, pit formation, and F-actin ring staining assays in RAW 264.7 cells treated with RANKL with or without melittin ( ${ }^{*}<0.01$ compared to non-treated cells) (E) The assessment of osteoclast-like cells differentiated from bone marrow-derived macrophages (BMMs) was done using TRAP staining and F-actin ring staining assays. Data are representative of 3 independent experiments. 
A

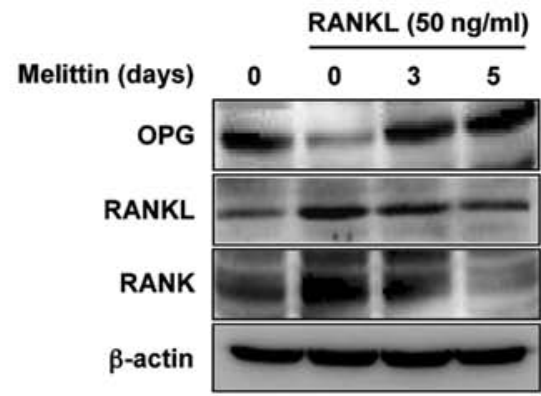

\begin{tabular}{|c|c|c|c|c|c|}
\hline & \multicolumn{3}{|c|}{ Culture medium } & \multicolumn{2}{|c|}{ Melittin $(1 \mu \mathrm{g} / \mathrm{ml})$} \\
\hline & 0 & 15 & 30 & 0 & 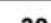 \\
\hline
\end{tabular}

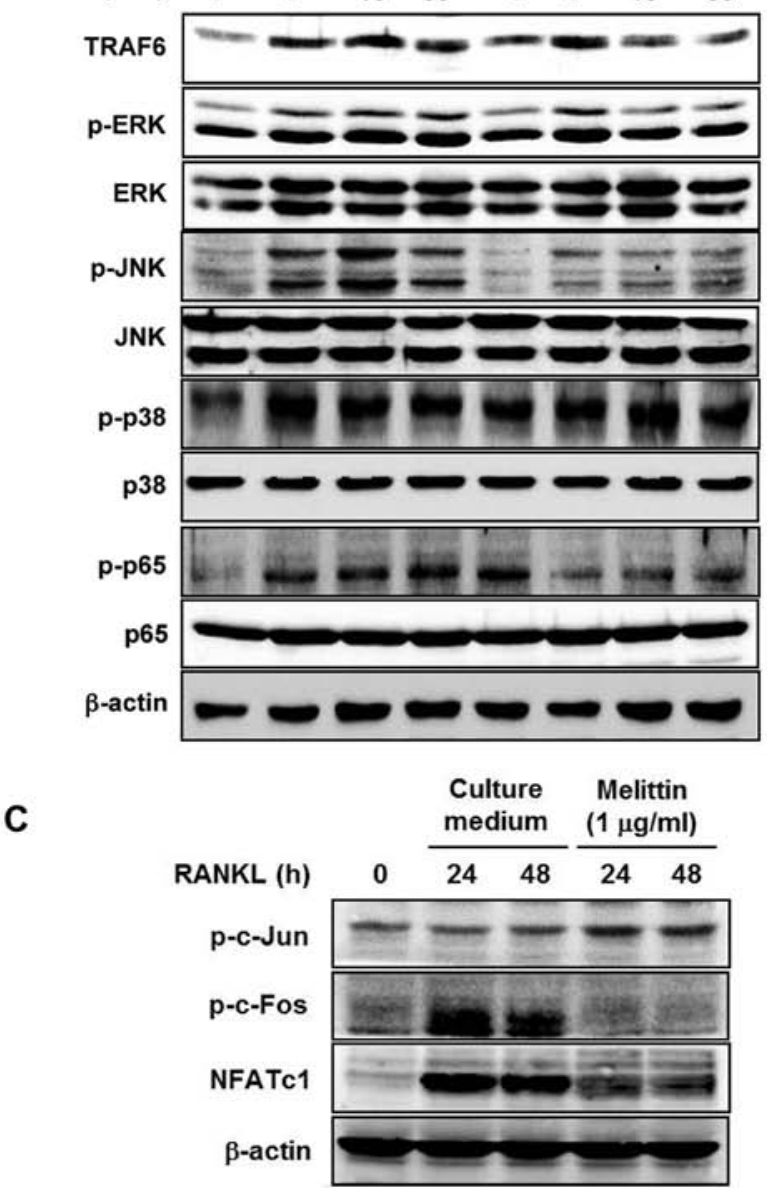

Figure 3. Osteoclast formation is regulated through the activation of mitogenactivated protein kinases (MAPKs) and their downstream molecules. (A) Crucial molecules involved in osteoclastogenesis, such as osteoprotegerin (OPG), receptor activator of nuclear factor- $\kappa$ B ligand (RANKL), and RANK, in RAW 264.7 cells cultured with RANKL were time-dependently assessed after melittin treatment $(1 \mu \mathrm{g} / \mathrm{ml})$. (B) At the downstream of the RANKL-RANK system, the expression of TNF receptor-associated factor-6 (TRAF6) and MAPKs in culture medium-treated cells and melittintreated cells was measured. (C) Phosphorylation of c-Jun, c-Fos and nuclear factor of activated T cells cytoplasmic 1 (NFATc1) in RAW 264.7 cells cultured with RANKL was assessed according to treatment with and without melittin by western blot analysis. Data are representative of 3 independent experiments.

stimualted with RANKL (50 ng/ml) and M-CSF (30 ng/ml) for 5 days differentiated into osteoclast-like MNCs, as shown by the increased number of TRAP $(+)$ cells; this effect was significantly suppressed by treatment with melittin $(1 \mu \mathrm{g} / \mathrm{ml})$. The F-actin ring formation assay also revealed larger and numerous F-actin ring(+) MNCs following stimulation with RANKL, and this effect was also significantly suppressed by treatment with melittin (1 $\mu \mathrm{g} / \mathrm{ml})$ (Fig. 2E).

Melittin regulates the activation of MAPKs, $c-F o s$ and NFATcl in RANKL-induced osteoclastogenesis. After stimulating the RAW 264.7 cells with RANKL (50 ng/ml), OPG protein expression was markedly decreased compared to the levels in the non-stimulated RAW 264.7 cells (Fig. 3A). By contrast, there was an increased protein expression of RANKL and RANK in the RAW 264.7 cells stimulated with RANKL. However, melittin $(1 \mu \mathrm{g} / \mathrm{ml})$ enhanced $\mathrm{OPG}$ protein expression in a time-dependent manner, whereas it decreased the protein expression of RANKL and RANK.

The protein expression of TRAF6, p-ERK, p-JNK and p-p65 in the RAW 264.7 cells stimulated with RANKL $(50 \mathrm{ng} / \mathrm{ml})$ which was increased by RANKL at 15 and 30 min was markedly attenuated by pre-treatment with melittin $(1 \mu \mathrm{g} / \mathrm{ml})$ for $2 \mathrm{~h}$, in comparison to the levels in culture medium-treated cells; however, the protein level of p-p38 was not altered by melittin treatment (Fig. 3B). In the assessment of the downstream of RANK-RANKL system in osteoclastogenesis, the results of western blot analysis revealed that the increased p-c-Fos and NFATc1 expression induced by RANKL in the RAW 264.7 cells was prominently suppressed by treatment with melittin $(1 \mu \mathrm{g} / \mathrm{ml})$; however, the phosphorylation of c-Jun was not significantly affected (Fig. 3C).

IL-1 $\beta$ has a crucial effect on the formation of osteoclast-like $M N C s$. This experiment was designed to assess whether IL-1 $\beta$ contributes to the development of mature osteoclasts. Fig. 4A illustrates that the mRNA expression of IL-1 $\beta$ in the RAW 264.7 cells stimulated with RANKL increased in a time-dependent manner. In addition, the RANKL-stimulated RAW 264.7 cells exhibited a trend towards an increased TNF- $\alpha$ mRNA expression. However, treatment of the BMMs with M-CSF alone or M-CSF combined with IL-1 $\beta(100 \mathrm{ng} / \mathrm{ml})$ did not induce the formation of osteoclast-like MNCs, as shown by TRAP and F-actin ring assays (Fig. 4B). The number of TRAP(+) osteoclast-like MNCs derived from the BMMs treated together with M-CSF, RANKL and IL- $1 \beta$ increased in comparison to the BMMs treated with M-CSF and RANKL (p<0.05; Fig. 4B). Consistently, we observed an increased TRAP gene expression in BMMs treated together with M-CSF, RANKL and IL-1 $\beta$, compared to that in the cells treated with M-CSF and RANKL (p<0.05; Fig. 4C).

To confirm the synergistic effect of IL-1 $\beta$ and RANKL on osteoclast formation, the presence of TRAP $(+)$ and F-actin ring(+) osteoclast-like MNCs was detected in the control cells and IL-1 $\beta$ siRNA-transfected cells stimulated with RANKL alone (Fig. 4D). Few osteoclast-like MNCs were detected in the RAW 264.7 cells transfected with IL-1 $\beta$ siRNA $(\mathrm{p}<0.001$; Fig. 4D). The increased expression of TRAP $(p<0.001)$ and cathepsin $\mathrm{K}(\mathrm{p}<0.05)$ genes induced by RANKL was significantly suppressed in the RAW 264.7 cells transfected with IL-1 $\beta$ siRNA (Fig. 4E).

Melittin synergistically attenuates the effects of RANKL on osteoclastogenesis in the cells transfected with $I L-1 \beta$ siRNA. The increased protein expression of IL-1 $\beta$ in the cells not treated with melittin and stimulated with RANKL was 

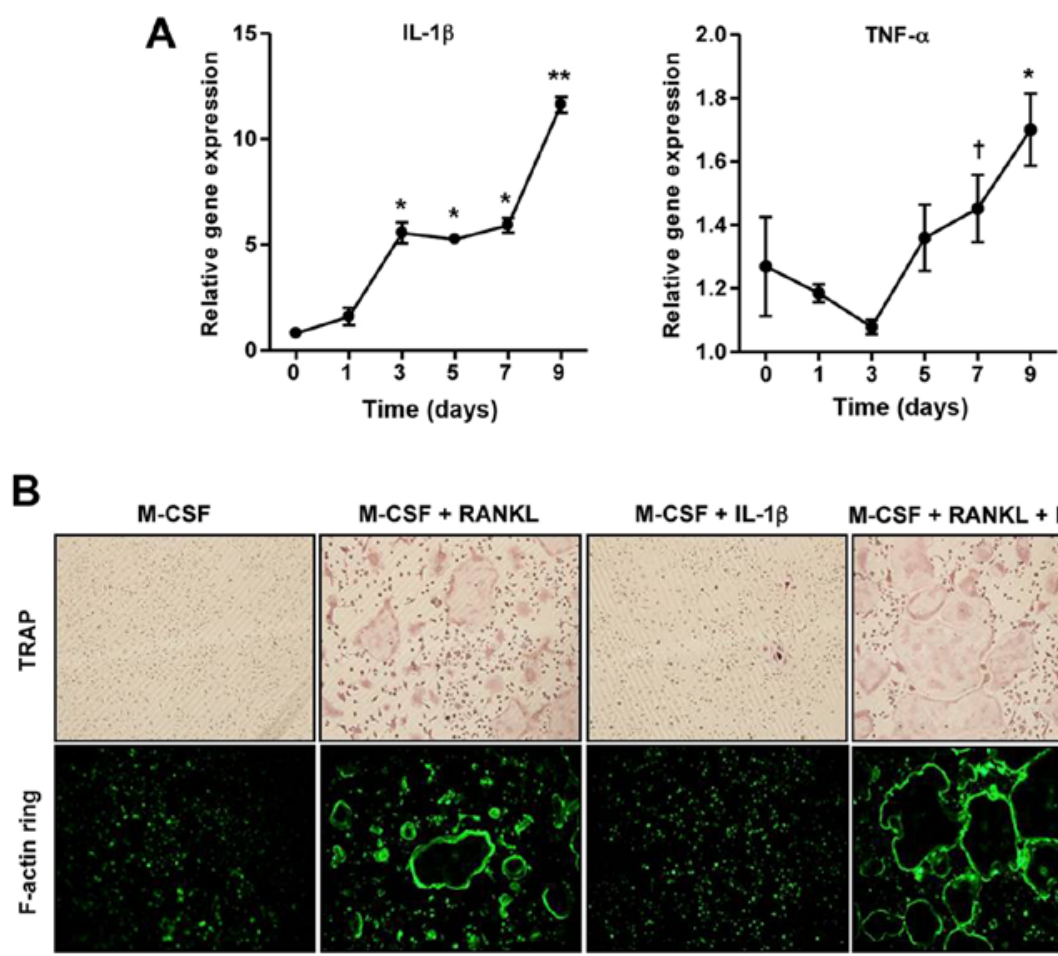

M-CSF + RANKL

$\mathrm{M}-\mathrm{CSF}+\mathrm{IL}-1 \beta$

M-CSF + RANKL + IL-1 $1 \beta$
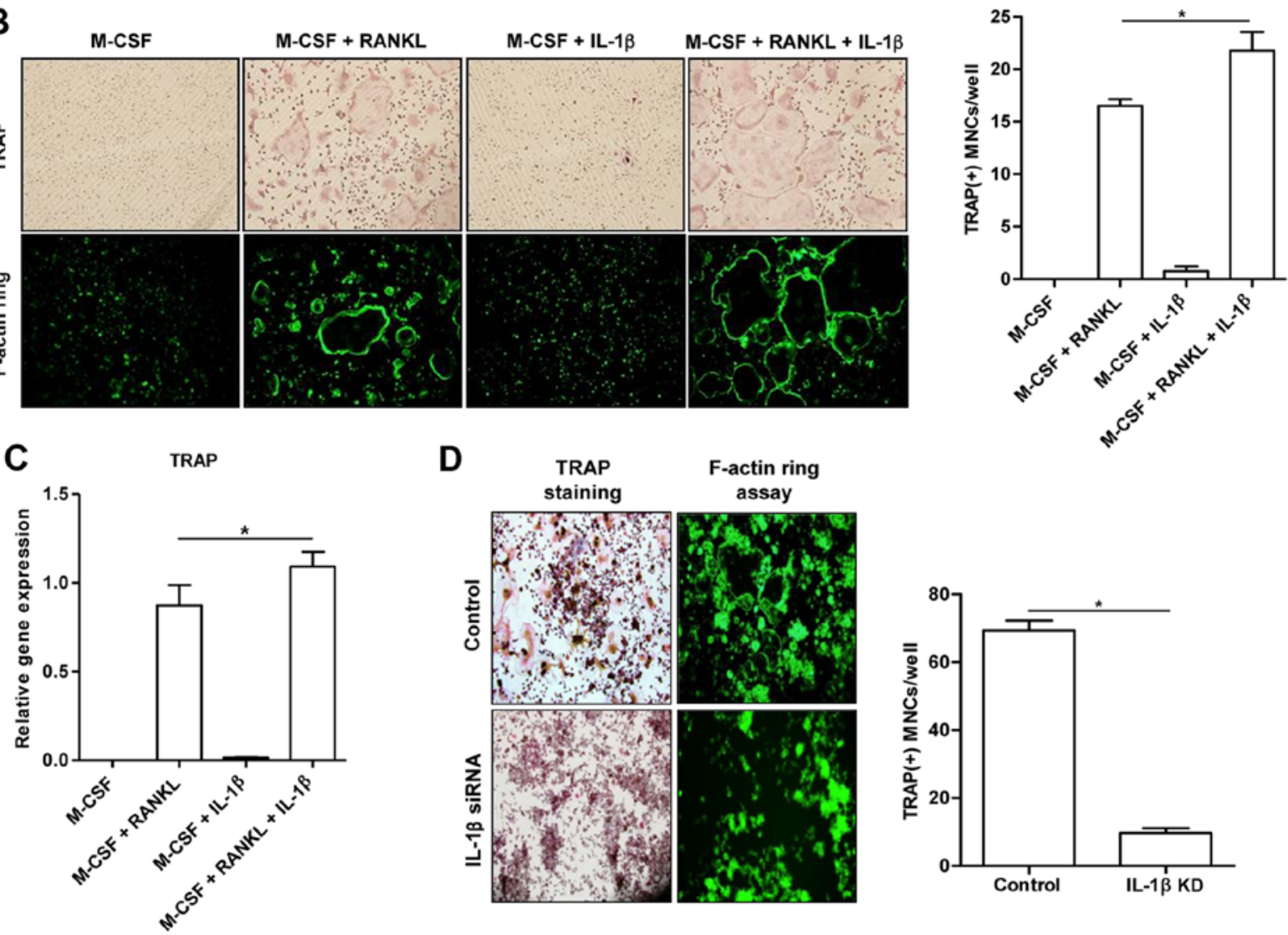

E
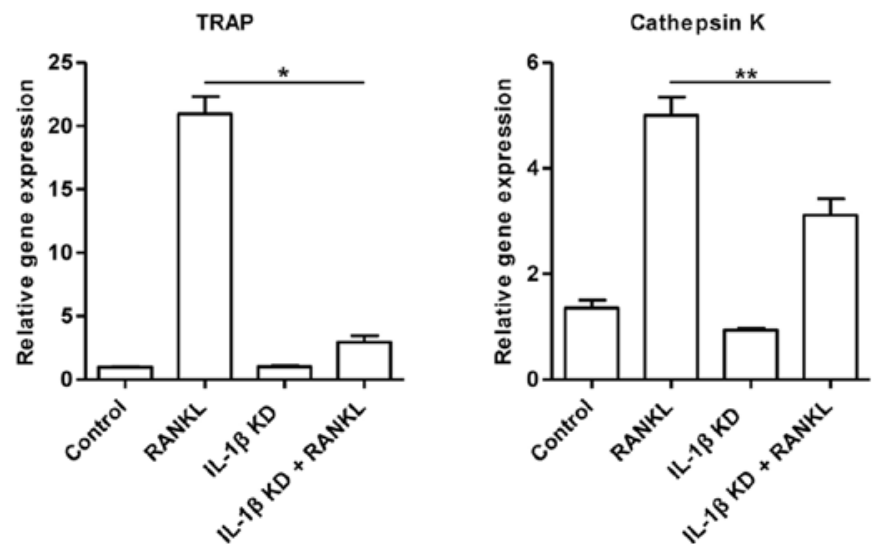

Figure 4. Interleukin-1 $\beta$ (IL-1 $\beta$ ) is involved in the formation of osteoclast-like MNCs. (A) IL-1 $\beta$ and tumor necrosis factor- $\alpha$ (TNF- $\alpha$ ) expression in RAW 264.7 cells cultured with receptor activator of nuclear factor- $\mathrm{kB}$ ligand (RANKL) were measured in over a period of time ( ${ }^{*} \mathrm{p}<0.01,{ }^{* *} \mathrm{p}<0.001$ and ${ }^{\dagger} \mathrm{p}<0.05$ compared to non-treated cells). (B) The in vitro morphological and functional analyses for osteoclastogenesis were performed using TRAP staining and F-actin ring staining assays in bone marrow-derived macrophages (BMMs) cultured with macrophage colony-stimulating factor (M-CSF), RANKL, or IL-1 $\beta$ ("p $<0.05$ compared to cells treated together with M-CSF and RANKL). (C) TRAP gene expression was assessed in BMM cells cultured with M-CSF, RANKL or IL-1 $\beta$ ("p $<0.05$ compared to cells treated together with M-CSF and RANKL). (D) The in vitro morphological and functional analyses for osteoclastogenesis were performed using TRAP staining and F-actin ring staining assays in RAW 264.7 cells transfected with IL-1 $\beta$ siRNA and control cells (" $\mathrm{p}<0.001$ compared to control cells). (E) The expression of TRAP and cathepsin K genes induced by RANKL was measured in RAW 264.7 cells transfected with IL-1 $\beta$ siRNA and control cells ("p $<0.001$ and ${ }^{* *} \mathrm{p}<0.05$ compared to RANKL-stimulated cells). Data are representative of 3 independent experiments. IL-1 $\beta$ KD, IL-1 $\beta$ knockdown by siRNA. 
A

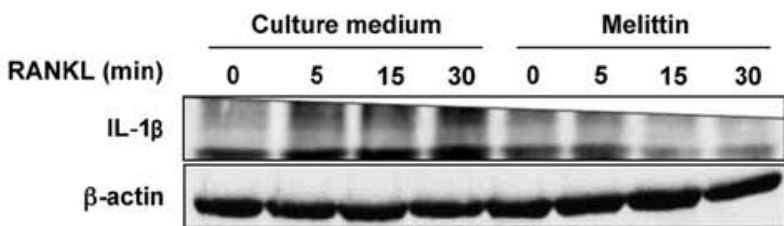

B

B Culture medium
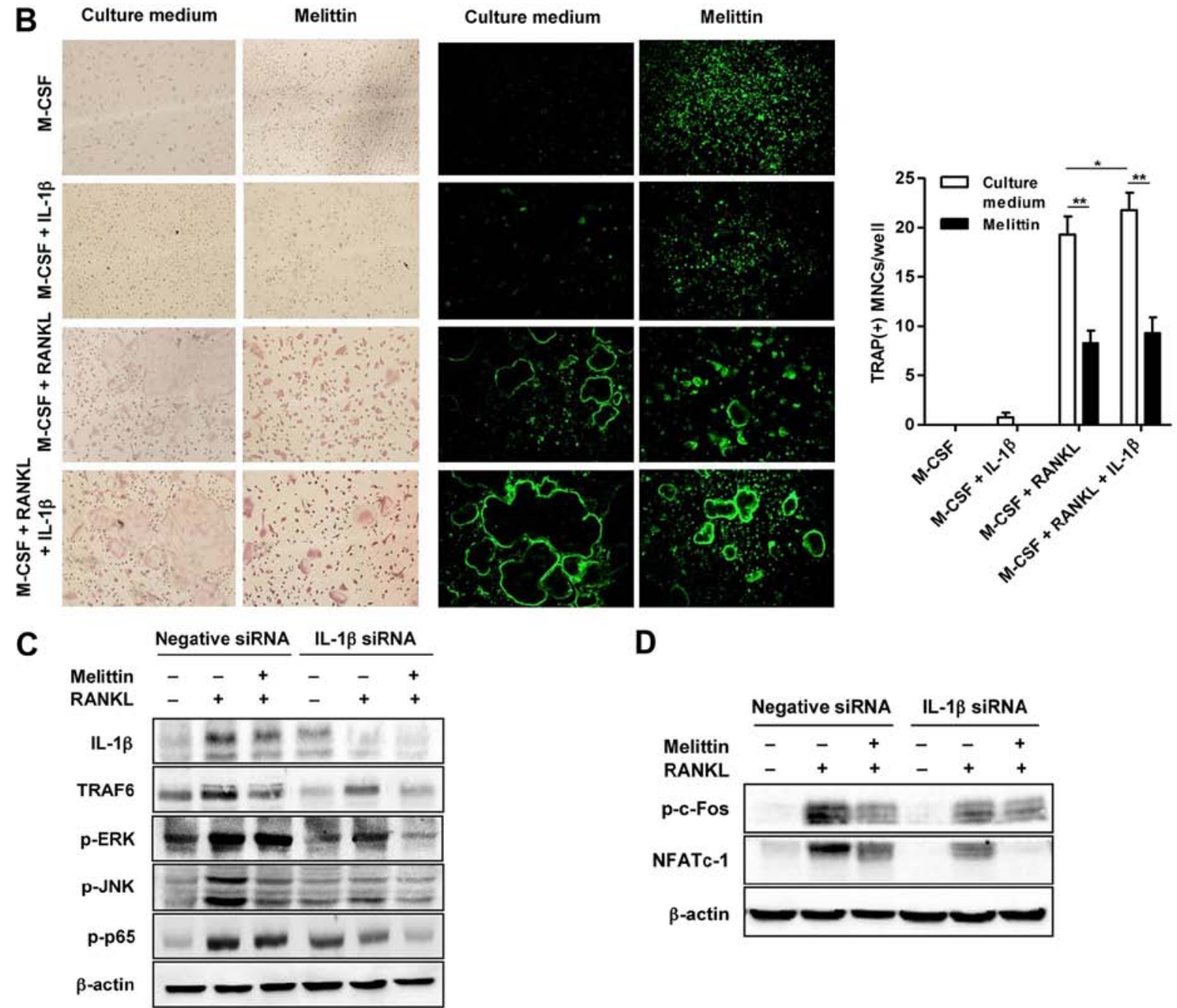

D

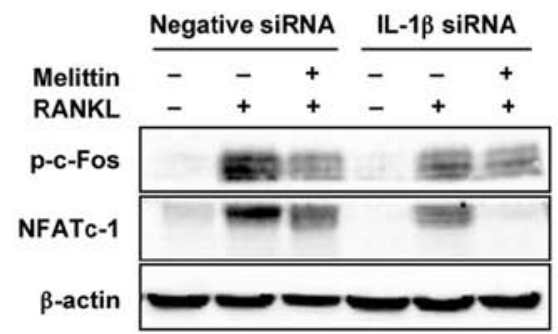

Figure 5. Melittin reduces interleukin-1 $\beta$ (IL-1 $\beta$ )-mediated osteoclastogenesis. (A) Receptor activator of nuclear factor-kB ligand (RANKL)-induced IL-1 $\beta$ production in RAW 264.7 cells treated with and without melittin was measured. (B) The in vitro morphological and functional analyses for osteoclastogenesis were performed using TRAP staining and F-actin ring staining assays in bone marrow-derived macrophages (BMMs) cultured with macrophage colony-stimulating factor (M-CSF), RANKL, IL-1 $\beta$ or melittin ("p $<0.05$ compared to culture medium-treated cells treated with M-CSF and RANKL and ${ }^{* *} \mathrm{p}<0.01$ compared to each culture medium-treated cell). (C) Production of IL-1 $\beta$, TNF receptor-associated factor-6 (TRAF6), p-ERK, p-JNK and p-p65 was measured in RAW 264.7 cells transfected with IL-1 $\beta$ siRNA and negative siRNA, under stimulation of melittin or RANKL. (D) Production of p-c-Fos and nuclear factor of activated T cells cytoplasmic 1 (NFATc1) was measured in RAW 264.7 cells transfected with IL-1 $\beta$ siRNA and negative siRNA, under stimulation of melittin or RANKL. Data are representative of 3 independent experiments.

noted, as shown by western blot analysis (Fig. 5A). By contrast, melittin markedly suppressed IL-1 $\beta$ protein expression in the RANKL-stimulated RAW 264.7 cells (Fig. 5A). Analysis using TRAP staining and F-actin staining revealed numerous TRAP(+) and F-actin ring(+) osteoclast-like MNCs in the BMMs co-stimulated with RANKL $(50 \mathrm{ng} / \mathrm{ml})$ and M-CSF (30 ng/ml) (Fig. 5B). The addition of IL-1 $\beta(100 \mathrm{ng} / \mathrm{ml})$ was shown to have a synergistic effect on osteoclastogenesis, illustrated by the more frequent detection of larger TRAP $(+)$ and F-actin ring(+)osteoclast-like MNCs compared to those in the BMMs stimulated with M-CSF and RANKL, but without IL-1 $\beta$. Consistently, the increased number of TRAP(+) MNCs in the BMMs co-stimulated with RANKL and M-CSF, with or without IL-1 $\beta$, was attenuated by melittin treatment (Fig. 5B).

RANKL induced an increase in the expression of IL-1 $\beta$, TRAF6, p-ERK, p-JNK and NF- $\mathrm{B}$ p65 in the RAW 264.7 cells transfected with negative siRNA, and this effect was significantly attenuated in the cells transfected with IL-1 $\beta$ siRNA (Fig. 5C). Treatment with melittin exerted a suppressive effect on the protein expression of TRAF6, p-JNK and p-p65 in the RAW 264.7 cells transfected with negative siRNA. In the RAW 264.7 cells transfected with IL-1 $\beta$ siRNA, melittin treatment also additionally inhibited the expression of these proteins, including TRAF6, p-ERK and p-p65. 


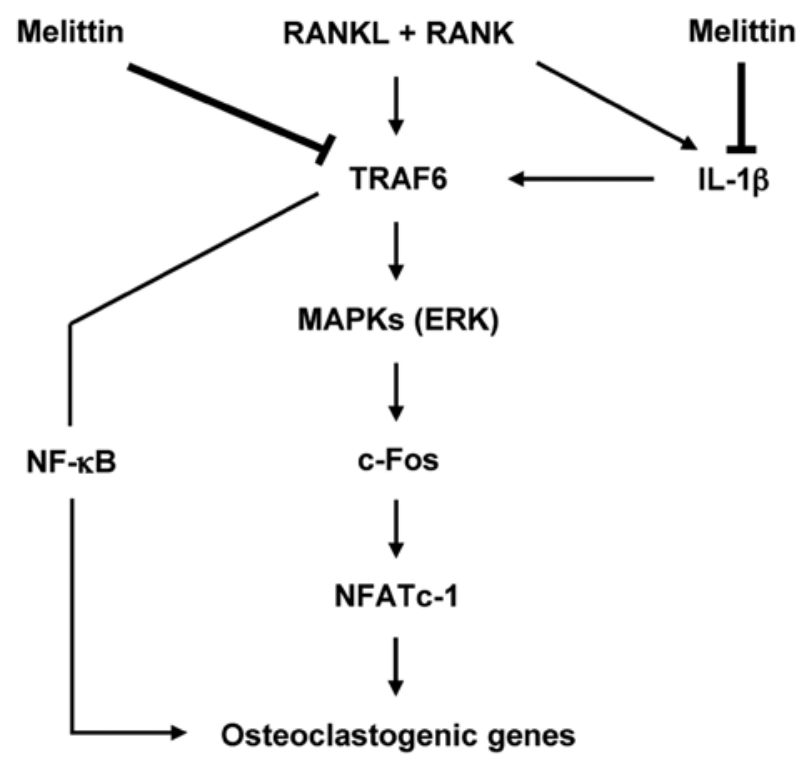

Figure 6. Proposed hypothesis for the effect of melittin on formation of osteoclast-like MNCs. Stimulation of receptor activator of nuclear factor- $\mathrm{\kappa}$ ligand (RANKL) to macrophages activates an adaptor protein TNF receptor-associated factor-6 (TRAF6) in the cytoplasm, sequentially mediates induction of phosphorylation of mitogen-activated protein kinases (MAPKs) particularly ERK, together with activation of NF- $\kappa \mathrm{B}$. Expression of osteoclastogenic genes including TRAP, cathepsin K, matrix metalloproteinase-9 (MMP-9), and carbonic anhydrase II was induced through phosphorylation of c-Fos and nuclear factor of activated $\mathrm{T}$ cells cytoplasmic 1 (NFATc1) at the downstream in signal transduction of MAPK pathway. In addition, the stimulation of macrophages with RANKL induces an increase in interleukin-1 $\beta$ (IL-1 $\beta$ ) expression, which may contribute to osteoclastogenesis. These signals are effectively inhibited by melittin targeting TRAF6 and IL-1 $\beta$.

In addition, stimulation with RANKL increased p-c-Fos and NFATc1 expression in the RAW 264.7 cells transfected with negative siRNA; this effect was markedly suppressed in the cells transfected with IL-1 $\beta$ siRNA (Fig. 5D). Melittin synergistically inhibited NFATc1 expression in the RAW 264.7 cells transfected with IL-1 $\beta$ siRNA (Fig. 5D).

\section{Discussion}

Osteoclast differentiation and activation are mainly regulated by the RANK-RANKL system $(1,2)$. A pathogenic role for osteoclast differentiation and activation has been noted in conditions of chronic inflammatory arthritis, including RA, PsA and gouty arthritis, as diverse pro-inflammatory cytokines trigger bone erosion upon the activation of the RANK-RANKL system (4-7). In particular, melittin has shown to have potent anti-arthritic properties in in vitro and in vivo studies, as indicated by the attenuation of inflammatory responses through the inhibition of $\mathrm{NF}-\kappa \mathrm{B}$ activation and the induction of the apoptosis of fibroblast-like synoviocytes and murine or human macrophages $(14,16)$. Based on these data, we hypothesized that melittin has the potential to protect against bone erosion by blocking osteoclast formation in inflammatory conditions. To the best of our knowledge, to date, there is no evidence of the effect of melittin on osteoclast formation at the molecular level. In the present study, we revealed that melittin inhibited osteoclast formation in RAW 264.7 cells and BMMs through the inhibition of the RANKL-RANK system and of the osteoclastogenic effect of IL-1 $\beta$.
Melittin, a major component of whole bee venom, is a small protein with 26 amino acid residues and a water-soluble tetramer that acts as a lytic agent (15). Whole bee venom is composed of $90 \%$ water-soluble, $5 \%$ ethyl acetate-soluble and $5 \%$ hexanesoluble components (13). Bee venom has been found to exert an anti-arthritic effect through the attenuation of inflammatory responses in rat models of adjuvant-induced $(13,14)$ and type II collagen-induced arthritis (19). Kwon et al demonstrated that treatment with the water-soluble fraction of bee venom significantly suppressed pathological bone changes in affected joints, whereas the ethyl acetate fraction of bee venom did not interfere with bone damage to arthritic joints (13). They proposed that melittin may be a potent candidate for anti-inflammatory therapeutics in arthritis as it is a main component of the water-soluble fraction of bee venom. By contrast, Tashjian et al reported that melittin was a stimulator of prostaglandin $\mathrm{E}_{2}\left(\mathrm{PGE}_{2}\right)$ synthesis and bone resorption in neonatal mouse calvaria (17). In the present study, the increased gene expression of osteoclast markers induced by RANKL, such as TRAP, cathepsin K, MMP-9 and carbonic anhydrase II, was significantly attenuated by melittin treatment in RAW 264.7 cells. In addition, melittin was found to inhibit the formation of osteoclast-like MNCs, as shown by TRAP staining and F-actin ring assays in RAW 264.7 cells and BMMs. This evidence indicates that melittin inhibits the formation of osteoclast-like MNCs differentiated from macrophages, which is in accordance with the findings of a previous study (13).

RANK is a member of the TNF receptor (TNFR) superfamily, which sequentially recruits TRAFs $(1,2)$. TRAF proteins are adaptor proteins which play an important role in signal transduction after the binding of RANK-RANKL. Among the TRAF proteins, TRAF6 seems to be important in RANKL-induced osteoclastogenesis, as shown by the defective activation of osteoclasts differentiated from osteoclast precursor cells in $\mathrm{TRAF}^{-/-}$mice (20). TRAF6 mediates the activation of RANKL-induced NF- $\kappa \mathrm{B}$ and MAPK signaling, which have been well recognized as downstream targets in osteoclastogenesis $(1,2)$. RANKL, through the degradation of $\mathrm{I} \kappa \mathrm{B} \alpha$, is involved in the activation of the NF- $\kappa \mathrm{B}$ dimer, p50/p65, in both BMMs and RAW 264.7 cells (21). ERK activity in osteoclast-like cells has been found to be closely related to osteoclast survival, but not bone resorption by the regulation of apoptosis (22). BMMs derived from mice which are $\mathrm{JNK} 1^{-/}$, but not $\mathrm{JNK} 2^{-/}$, exhibit reduced differentiation into RANKL-induced osteoclasts (23). It has been well established that the $N F-\kappa B$ and MAPK signaling pathways are targets for inflammatory responses regulated by melittin (24). In the present study, we investigated whether melittin regulates the activation of the NF- $\kappa \mathrm{B}$ and MAPK cascades, and participates in the process of the formation of osteoclast-like MNCs. Our results demonstrated that melittin treatment inhibited the increased protein expression of TRAF6, p-ERK, p-JNK and $\mathrm{NF}-\kappa \mathrm{B}$ (p65) induced by RANKL in RAW 264.7 cells, but not that of p-p38 MAPK.

The dimeric AP-1 transcription factor in osteoclastogenesis is a downstream target of the ERK and JNK pathway $(1,2)$. In addition, NFATc1 is also considered a transcriptional regulator of osteoclastogenesis, which can be activated by ERK. ERK activates AP-1 transcriptional activity by the phosphorylation of c-Fos, while c-Jun activation is induced by JNK. In this 
study, we found that RANKL-induced c-Fos, and NFATc1 were activated in RAW 264.7 cells, but not c-Jun. Sequentially, melittin treatment was shown to inhibit the phosphorylation of c-Fos and NFATc1. This suggests that downstream ERK in signal transduction may be major determinant during osteoclastogenesis.

IL-1 $\beta$ is a multifunctional cytokine that targets various cells and tissues. In bone metabolism, IL-1 $\beta$ is known to be responsible for each step of osteoclast-induced bone resorption, such as differentiation and activation $(9,10,25)$. In addition, this cytokine plays an important role in increasing osteoclast survival time, which may be due to IL-1 $\beta$ working together with RANKL to induce NF- $\kappa \mathrm{B}$ activation (25). Tanabe et al showed that IL-1 alone could not drive osteoclast formation (26). By contrast, Kim et al demonstrated that IL-1 alone directly induced osteoclast differentiation if the IL-1 receptor was expressed on osteoclast precursor cells, such as BMMs, in the RANKLdependent or RANKL-independent pathway (9). Anakinra, a recombinant human IL-1 receptor antagonist, was shown to significantly reduce radiographic joint erosion in RA (11). In this study, we used BMMs to identify a synergistic role for IL-1 $\beta$ in the formation of osteoclast-like MNCs. This was supported by the decreased expression of osteosclast-specific genes, such as TRAP and cathepsin K, and decreased osteoclast-like MNCs formation in RAW 264.7 cells lacking IL-1 $\beta$. Melittin has the potential to suppress IL-1 expression, as illustrated through the blocked activation of the NF- $\kappa \mathrm{B}$ and MAPK pathway by exposure to Propionibacterium acnes in HaCaT cells (24), although Stuhlmeier demonstrated that neither BV nor melittin blocked IL-1 $\beta$ expression in experimental cells (27). This study also revealed that melittin inhibited endogenous IL-1 $\beta$ production in RANKL-stimulated cells.

Our results suggest that the effect of melittin on RANKL-induced osteoclast formation may be dependent on the activation of c-Fos and NFATc1 downstream of MAPKs, particularly ERK, as these signal transduction molecules were assessed to delineate the mechanism of IL-1 $\beta$ on osteoclast-like MNC formation. This study demonstrated that the activation of NFATc1 and the phosphorylation of NF- $\kappa$ B, ERK, JNK and c-Fos were markedly attenuated in IL- $1 \beta$ siRNA transfected RAW 264.7 cells. In particular, c-Fos may be a crucial component in osteoclastogenesis, either by a RANKL-dependent or RANKL-independent mechanism (9). The induction of the IL-1 receptor by overexpression of c-Fos could contribute to the formation of osteoclast-like cells stimulated by IL-1 alone in a RANKL-independent mechanism.

In conclusion, the importance of this study is that melittin has the ability to potently suppress osteoclast-like MNC formation through the inhibition of c-Fos and NFATc1 downstream of the MAPK signaling pathway in osteoclastogenesis (Fig. 6). It is also worth noting that IL- $1 \beta$ is an important cytokine for regulating osteoclast-like MNC formation, and may be considered as a promising therapeutic target for bone loss or erosion in chronic inflammatory arthritis.

\section{Acknowledgements}

The present study was supported by the Daegu Catholic University Medical Center Regional Center for Rheumatic Diseases and Degenerative Arthritis research grant. We would like to thank Dr Ki-Yeun Park for technical assistance of TRAP and $\mathrm{F}$-actin ring assays.

\section{References}

1. Takayanagi H: Osteoimmunology: Shared mechanisms and crosstalk between the immune and bone systems. Nat Rev Immunol 7: 292-304, 2007.

2. Asagiri $\mathrm{M}$ and Takayanagi $\mathrm{H}$ : The molecular understanding of osteoclast differentiation. Bone 40: 251-264, 2007.

3. Teitelbaum SL: Osteoclasts; culprits in inflammatory osteolysis. Arthritis Res Ther 8: 201, 2006.

4. Udagawa N, Kotake S, Kamatani N, Takahashi N and Suda T: The molecular mechanism of osteoclastogenesis in rheumatoid arthritis. Arthritis Res 4: 281-289, 2002.

5. Colucci S, Brunetti G, Cantatore FP, Oranger A, Mori G Quarta L, Cirulli N, Mancini L, Corrado A, Grassi FR and Grano M: Lymphocytes and synovial fluid fibroblasts support osteoclastogenesis through RANKL, TNF $\alpha$, and IL-7 in an in vitro model derived from human psoriatic arthritis. J Pathol 212: 47-55, 2007.

6. Dalbeth N, Smith T, Nicolson B, Clark B, Callon K, Naot D, Haskard DO, McQueen FM, Reid IR and Cornish J: Enhanced osteoclastogenesis in patients with tophaceous gout: Urate crystals promote osteoclast development through interactions with stromal cells. Arthritis Rheum (Munch) 58: 1854-1865, 2008.

7. Lee SJ, Nam KI, Jin HM, Cho YN, Lee SE, Kim TJ, Lee SS Kee SJ, Lee KB, Kim N and Park YW: Bone destruction by receptor activator of nuclear factor $\kappa \mathrm{B}$ ligand-expressing $\mathrm{T}$ cells in chronic gouty arthritis. Arthritis Res Ther 13: R164, 2011.

8. Gowen M, Wood DD, Ihrie EJ, Meats JE and Russell RG: Stimulation by human interleukin 1 of cartilage breakdown and production of collagenase and proteoglycanase by human chondrocytes but not by human osteoblasts in vitro. BBA 797: 186-193, 1984.

9. Kim JH, Jin HM, Kim K, Song I, Youn BU, Matsuo K and Kim N: The mechanism of osteoclast differentiation induced by IL-1. J Immunol 183: 1862-1870, 2009.

10. Wei S, Kitaura H, Zhou P, Ross FP and Teitelbaum SL: IL-1 mediates TNF-induced osteoclastogenesis. J Clin Invest 115: 282-290, 2005.

11. Strand V and Kavanaugh AF: The role of interleukin-1 in bone resorption in rheumatoid arthritis. Rheumatology (Oxford) 43 (Suppl 3): iii10-iii16, 2004.

12. Billingham ME, Morley J, Hanson JM, Shipolini RA and Vernon CA: Letter: An anti-inflammatory peptide from bee venom. Nat Lett 245: 163-164, 1973.

13. Kwon YB, Lee HJ, Han HJ, Mar WC, Kang SK, Yoon OB, Beitz AJ and Lee JH: The water-soluble fraction of bee venom produces antinociceptive and anti-inflammatory effects on rheumatoid arthritis in rats. Life Sci 71: 191-204, 2002.

14. Park HJ, Lee SH, Son DJ, Oh KW, Kim KH, Song HS, Kim GJ, Oh GT, Yoon DY and Hong JT: Antiarthritic effect of bee venom: Inhibition of inflammation mediator generation by suppression of NF- $\kappa \mathrm{B}$ through interaction with the p50 subunit. Arthritis Rheum (Munch) 35: 3504-3515, 2004.

15. Terwilliger JT and Eisenberg D: The structure of melittin. II. Interpretation of the structure. J Biol Chem 257: 60166022, 1982.

16. Kim SK, Park KY, Yoon WC, Park SH, Park KK, Yoo DH and Choe JY: Melittin enhances apoptosis through suppression of IL-6/sIL-6R complex-induced NF- $\kappa$ B and STAT3 activation and $\mathrm{Bcl}-2$ expression for human fibroblast-like synoviocytes in rheumatoid arthritis. Joint Bone Spine 78: 471-477, 2011.

17. Tashjian AH Jr, Ivey JL, Delclos B and Levine L: Stimulation of prostaglandin production in bone by phorbol diesters and melittin. Prostaglandins 16: 221-232, 1978.

18. Park H,Jung YK, Park OJ,Lee YJ, Choi JY and Choi Y: Interaction of Fas ligand and Fas expressed on osteoclast precursors increases osteoclastogenesis. J Immunol 175: 7193-7201, 2005.

19. Lee JD, Kim SY, Kim TW, Lee SH, Yang HI, Lee DI and Lee YH: Anti inflammatory effect of bee venom on type II collagen-induced arthritis. Am J Chin Med 32: 361-367, 2004.

20. Naito A, Azuma S, Tanaka S, Miyazaki T, Takaki S, Takatsu K, Nakao K, Nakamura K, Katsuki M, Yamamoto T and Inoue J: Severe osteopetrosis, defective interleukin-1 signalling and lymph node organogenesis in TRAF6-deficient mice. Genes Cells 4: 353-362, 1999. 
21. Wei S, Teitelbaum SL, Wang MW and Ross FP: Receptor activator of nuclear factor- $\kappa \mathrm{B}$ ligand activates nuclear factor- $\kappa \mathrm{B}$ in osteoclast precursors. Endocrinology 142: 1290-1295, 2001.

22. Miyazaki T, Katagiri H, Kanegae Y, Takayanagi H, Sawada Y, Yamamoto A, Pando MP, Asano T, Verma IM, Oda H, et al: Reciprocal role of ERK and NF- $\kappa \mathrm{B}$ pathways in survival and activation of osteoclasts. J Cell Biol 148: 333-342, 2000.

23. David JP, Sabapathy K, Hoffmann O, Idarraga $\mathrm{MH}$ and Wagner EF: JNK1 modulates osteoclastogenesis through both c-Jun phosphorylation-dependent and -independent mechanisms. J Cell Sci 115: 4317-4325, 2002.

24. Lee WR, Kim KH, An HJ, Kim JY, Chang YC, Chung H, Park YY, Lee ML and Park KK: The protective effects of melittin on Propionibacterium acnes-induced inflammatory responses in vitro and in vivo. J Invest Dermatol 134: 1922-1930, 2014.

25. Jimi E, Akiyama S, Tsurukai T, Okahashi N, Kobayashi K, Udagawa N, Nishihara T, Takahashi N and Suda T: Osteoclast differentiation factor acts as a multifunctional regulator in murine osteoclast differentiation and function. J Immunol 163 : 434-442, 1999
26. Tanabe N, Maeno M, Suzuki N, Fujisaki K, Tanaka H, Ogiso B and Ito K: IL-1a stimulates the formation of osteoclast-like cells by increasing $\mathrm{M}-\mathrm{CSF}$ and $\mathrm{PGE}_{2}$ production and decreasing OPG production by osteoblasts. Life Sci 77: 615-626, 2005.

27. Stuhlmeier KM: Apis mellifera venom and melittin block neither $\mathrm{NF}-\kappa \mathrm{B}-\mathrm{p} 50-\mathrm{DNA}$ interactions nor the activation of $\mathrm{NF}-\kappa \mathrm{B}$, instead they activate the transcription of proinflammatory genes and the release of reactive oxygen intermediates. J Immunol 179: 655-664, 2007. 